\title{
Current and new indications for corneal cross-linking in the therapy of corneal diseases
}

Katarzyna Przewłócka, Piotr Kanclerz

Hygeia Clinic, Gdańsk Head: Piotr Kanclerz MD, PhD

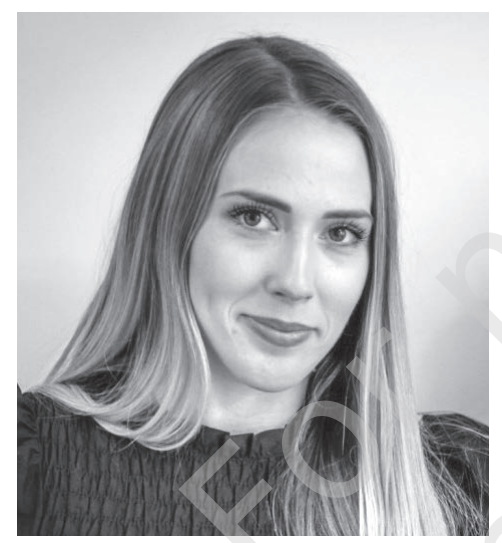

H I G H L I G H T S

Corneal collagen cross-linking is most often used in ectatic lesions, but it is also may be used in bacterial infections and bullous keratopathy. Corneal collagen cross-linking increases corneal stiffness, commonly improving visual acuity and topographical surface regularity.

\begin{abstract}
Corneal collagen cross-linking, a technique used in ophthalmology, involves the creation of new bonds between collagen fibers, thus leading to stabilization and corneal stiffening. The most common indication for corneal collagen cross-linking procedures is to prevent the progression of corneal ectasia, i.e., keratoconus, pellucid marginal degeneration, and ectasia after corneal refractive surgery. Clinical data also indicate that it is effective in the treatment of bacterial corneal infections and bullous keratopathy. Corneal collagen cross-linking was shown to inhibit the progression of corneal ectasia, and the treatment commonly leads to the improvement in visual acuity, decreases the maximum keratometry values, and improves the corneal topography image. In addition, it is possible to avoid or postpone the need for more complex and invasive surgery.
\end{abstract}

Key words: corneal collagen cross-linking, corneal ectasia, keratoconus, topography 


\section{INTRODUCTION}

Corneal cross-linking (CXL) is a surgical method used in the treatment of corneal ectasia [1]. Studies have shown a significant loss in the number of oblique connections of collagen fibers in corneal ectasia, which are responsible for maintaining the mechanical stability of the corneal structure and the eye surface [2]. Along with the loss of collagen fibers, destabilization of the cornea is observed, which results in irregular astigmatism, myopia, and deterioration of visual acuity.

CXL mechanism itself triggers a chemical reaction resulting in a formation of cross-links between individual proteins, initiated by ultraviolet radiation. By using riboflavin in combination with ultraviolet radiation, the formation of new bonds within the collagen fibers is induced. This results in strengthening of the collagen fibers' structure, which stabilizes and stiffens the surface of the cornea, while slowing or stopping the progress of the ectasia [1]. Furthermore, use of riboflavin that acts as a selective filter also protects deeper tissue structures against the harmful effects of UV rays [3]. Traditionally, treatment with CXL can be used for $400-\mu \mathrm{m}$ borderline thickness of the cornea, because complications related to damage to the endothelium and other structures of the eyeball can be avoided [4]. However, cross-linking procedures are now available for patients with a cornea thinner than $400 \mu \mathrm{m}$, using hypo-osmolar riboflavin solutions [5].

The first study of CXL was conducted by Wollensak et al., and the results were published in 2003 [6]. 23 patients participated in the study, which demonstrated the effectiveness of CXL in maintaining the stability of the corneal topography over a 4-year follow-up period [6]. Approximately $70 \%$ of patients experienced an improvement in keratometric values, while an improvement in corrected visual acuity was observed in $65 \%$ of tested patients. On the other hand, Caporossi et al. recorded a significant improvement in the observed topographical corneal morphology in $85 \%$ of subjects, and a decrease in the mean keratometry value with a simultaneous reduction in spherical equivalent refractive error [7, 8]. Similar observations were made by Asri et al., in a study on 42 eyes which showed a complete discontinuation of ectasia progression in $68.8 \%$ of subjects within 12 months [9]. At the same time, the maximum value of keratometry decreased by $2 \mathrm{D}$.

After CXL the following topical eye drops are used: antibiotics, glucocorticosteroids, and non-steroidal anti-inflammatory drugs. The use of a bandage contact lens is also recommended. A review of the available literature indicates a low incidence of complications after CXL. CXL is widely used in the world, and in Europe it has been recognized as one of the most effective and safe surgical procedures. After using CXL, the short-term occurrence of corneal stromal edema and a haze effect have so far been observed, howev- er, both of these conditions are usually transient [10]. Scientific reports suggest a higher risk of infectious keratitis in pregnant women for which an increased concentration of estrogen can negatively affect the biochemical stability of the cornea, causing its excessive stiffness and reducing the effects of the procedure [11].

\section{INDICATIONS FOR CROSS-LINKING}

\section{Keratoconus}

Keratoconus is a progressive, non-inflammatory eye disease leading to thinning and bulging of the cornea. The etiology of keratoconus is complex and both genetic and environmental factors predispose its occurrence. The risk factors include allergies, excessive eye rubbing, and frequent eyeball injuries [12]. This condition is also often associated with other diseases, such as Down's syndrome, Turner syndrome, mitral valve prolapse, connective tissue diseases and retinitis pigmentosa [13]. Keratoconus can significantly reduce visual acuity, distort the image, and increase sensitivity to light. In addition, these changes cannot be reversed by using corrective glasses due to the significant asymmetry of the cornea [14]. In Poland, keratoconus is currently the most common indication for corneal transplant surgery. For diagnosis of keratoconus, especially in its early stages, imaging techniques are used, such as corneal topography, corneal tomography, and optical coherence tomography of the anterior segment of the eye [16]. The main risk factors for the progression of keratoconus are the patient's young age at the time of diagnosis, and a maximum keratometry value above $55 \mathrm{D}$ [15]. In the majority of patients diagnosed with keratoconus at the age of 40, disease progression is not expected, however a diagnosis in late adolescence or early adulthood will most possibly mean deterioration of the topographic image within the next 12 months (tab. 1).

\section{TABLE}

Estimated changes in the maximum value of keratometry in patients with keratoconus based on the research collected in the meta-analysis by Ferdi et al. (based on [15]).

\begin{tabular}{c|c}
$\begin{array}{c}\text { Patient age } \\
\text { [years] }\end{array}$ & $\begin{array}{c}\text { Mean increase in maximum keratometry } \\
\text { over the 12-month follow-up period [D] }\end{array}$ \\
\hline 10 & 2 \\
\hline 15 & 1,6 \\
\hline 20 & 1,2 \\
\hline 25 & 0,8 \\
\hline 30 & 0,4 \\
\hline 35 & 0
\end{tabular}


More recent studies have shown that progression of keratoconus can also occur in patients aged over 30. In a study by Gokul et al. on patients aged $38.45 \pm 12.86$, progression of the maximum keratometry was $0.3 \pm 1.21 \mathrm{D}$ during a follow-up period of at least 12 months [17]. Increased deterioration of topographic parameters was found in approximately $20 \%$ of patients aged over 40 . Therefore, regular ophthalmic control with corneal topography or tomography is important even in patients who did not qualify for CXL. Also, the risk of keratoconus progression is higher in Middle Eastern peoples, lower in Europeans, and lowest in Asian patients [15]. Another progression risk factor is a high spherical equivalent refractive error at diagnosis [18].

\section{Pellucid marginal degeneration}

Pellucid marginal degeneration is a progressive and usually non-inflammatory of corneal ectasia. The term pellucid refers to the clarity of the cornea, despite the presence of ectasia. A characteristic feature of the disease is a crescent-shaped peripheral thinning of the cornea with a marked increase in optical power over the cornea [19]. With regards to histopathology, the pellucid marginal degeneration is considered a form of keratoconus [20]. The disease affects both women and men, but it is most often diagnosed in men aged 30 to 50. The etiology of pellucid marginal degeneration has not been clearly explained. In the microscopic image, the central thickness of the cornea and the arrangement of collagen fibers are normal. However, thinning as well as changes in the collagen structure and the presence of histiocytes have been found in some peripherally located foci [21, 22].

Due to presence of advanced thinning and the significant bulging of the cornea in this area, treatment of pellucid marginal degeneration is difficult. Therapy in patients at an early stage and with a mild or moderate course mainly consists of using vision correction with glasses or stable-shape contact lenses [23]. CXL and the implantation of intrastromal corneal ring segments are used in surgical treatment and keratoplasty procedures in advanced stages of the disease.

CXL is a recognized method of treatment of pellucid marginal degeneration, both in clinical and histopathological terms [24]. A technical problem in the use of CXL in the treatment of pellucid marginal degeneration may include difficulties in decentralizing of the ultraviolet beam to obtain a desired effect on the peripheral part of the cornea [25]. In most cases, however, it is still possible to perform the procedure. In a study by Stojanović et al., on patients treated with CXL in combination with individually selected ablation, both visual, refractive, and topographic results improved, and the progression of keratectasia was inhibited in the 12-month follow-up period. The authors suggested that in some cases this method may postpone or eliminate the need for corneal transplantation [26]. Similar observations were made by Kymionis et al., who demonstrated the effectiveness of CXL in the treatment of the pellucid corneal degeneration in a 34-year-old patient [27]. That patient's one-year follow-up showed post-surgery improvement of uncorrected and corrected visual acuity. Additionally, the topography image in both eyes had also improved. The case described by Hassan et al. of CXL treatment in a 55-year-old man with advanced pellucid degeneration confirmed the effectiveness and safety of the procedure, indicating that the disease progression had stopped, and visual acuity improved. No intraoperative or postoperative complications were observed [28]. In conclusion, CXL should be considered in patients with pellucid marginal degeneration, especially if there is a proven deterioration in topographic parameters or visual acuity.

\section{CORNEAL ECTASIA FOLLOWING PHOTOKERATITIS REFRACTION SURGERY}

In 1998, the phenomenon of corneal ectasia after laser-assisted in situ keratomileusis (LASIK) was described for the first time. A progressive reduction in the thickness of the cornea was observed with a local increase in its optical power, and an accompanying decrease in corrected and uncorrected visual acuity [29]. Keratectasia often has a genetic basis, but LASIK procedures can accelerate the onset of its symptoms in affected people [30]. The incidence of ectasia has been estimated at $0.02-0.6 \%$, most of which was observed after procedures to form a flap (especially of a large thickness) [31]. The risk factors for ectasia after photokeratitis refraction procedures primarily includes a forme fruste keratoconus (FFK) $[32,33]$. In their study, Brenner et al. showed that approx. $75 \%$ of patients who developed ectasia after LASIK had a hidden form of keratoconus [34]. Similar observations were made by Tatar et al., but this number was much smaller in their studies and amounted to $21.4 \%$ [35]. The current risk of developing ectasia after photokeratitis refraction procedures is very low due to the improved imaging techniques of the posterior corneal surface and biomechanical properties of the cornea [36]. The study results achieved by Hafezi et al. demonstrated that CXL treatment can reverse the progressive iatrogenic keratectasia after LASIK, which manifests as a reduction in maximum keratometric values [37]. The changes are most likely related to the restoration of cornea biochemical stability after its cross-linking. Advantageous results were seen during the 25-month follow-up period.

\section{Infectious keratitis}

The pathophysiology of infectious keratitis is associated with an invasion of microorganisms accompanied by a mi- 
gration of immune cells and the triggering of an inflammatory response. Secretion of pro-inflammatory cytokines (including metalloproteinases) leads to degradation of the collagen fibers, which causes cellular death. As a result of these changes, there is a gradual thinning of the cornea. Even though the infection can be managed with antimicrobial therapy, structural damage persists for a long time. For this reason, in the treatment of infectious keratitis, it is extremely important to use combined anti-inflammatory and antimicrobial, as well as anti-collagenolytic therapeutic methods [38]. It seems that CXL can be effective in the treatment of corneal infection due to its high potential for rebuilding collagen fibers.

The effectiveness of CXL of infectious keratitis in primary and complementary treatment in the case of a lack of effect of pharmacotherapy has previously been described in the literature. A study by Iseli et al. showed that CXL treatment inhibited corneal softening and reduced inflammatory infiltration in four out of five patients with therapy-refractory infectious keratitis [39].

Similar observations were made by Sorkhabi et al. in a group of 10 patients with antibiotic treatment-resistant corneal ulcers. Researchers achieved corneal healing with accompanying vision improvement in 8 out of 10 patients after CXL [40]. Makdoumi et al. also provided scientific evidence confirming the effectiveness of CXL in the treatment of severe, infectious keratitis. All seven patients achieved complete epithelialization and cessation of corneal softening. Additionally, in six out of seven treated eyes, a reduction in subjective symptoms within 24 hours of the procedure have been reported [41]. The effectiveness of CXL as an adjunctive therapy in antibiotic-resistant microbial infection of the cornea has been documented by Shetty et al. Out of 15 patients who underwent 14-day antibiotic therapy before CXL, the infection's regression in nine of them was reported, as well as pain relief in all patients on day 1 after the surgery. Regression of symptoms was not observed in patients with deep stromal keratitis [42]. In light of the current literature, it seems that the treatment of infectious keratitis with CXL is an effective and safe therapeutic strategy. CXL seems to have great potential as an alternative adjunctive method in the treatment of bacterial corneal ulcers, especially in recurrent inflammations [38]. For fungal keratitis where infections are caused by Acanthamoeba and herpetic keratitis, the evidence of CXL's effectiveness is inconclusive $[43,44]$.

\section{Bullous keratopathy}

Bullous keratopathy is one of the corneal endothelial diseases, and pathological changes include endothelial pump, corneal edema, and visual acuity deterioration [45]. In the past, bullous keratopathy was the most common complication following cataract surgery. Currently, it is much more often caused by Fuchs dystrophy, post-trauma damage to the corneal endothelium or damage following implantation of anterior chamber lenses. Bullous keratopathy is the main cause of endothelial keratoplasty [46]. Based on scientific evidence, CXL is considered an alternative method of treatment, reducing corneal swelling, and improving visual acuity. This method can delay or eliminate the need for keratoplasty. An increase in the density of collagen fibers and pressure reduction inside the corneal stroma are mechanisms that have been considered to improve the local condition after CXL [47].

Most of the studies on the use of CXL in bullous keratopathy have looked at the form is develops after cataract surgery. A prospective study by Ghanem et al. showed significantly improved clarity and reduction in the corneal thickness, and in eyeball pain, in the $1^{\text {st }}$ month after corneal collagen cross-linking. However, the authors pointed to the presumed lack of long-term effect because the beneficial changes decreased within 6 months after the procedure [48]. Positive results for pachymetry and corneal clarity have been reported by Krueger et al. following CXL treatment. Best spectacle-corrected visual acuity improved from finger counting to 20/80, postponing corneal transplantation for 6 months [49]. Wollensak et al. also observed good results from CXL, indicating significant improvement in visual acuity, corneal thickness, and eye pain by patients [50]. In contrast, the results of a randomized clinical trial showed that the use of CXL in bullous keratopathy may have a beneficial short-term effect, but the authors considered that these benefits were unlikely to outweigh the risk of recurrent epithelial defects. The reduction in central corneal thickness was significantly higher in the CXL group compared to the control group at weeks $2^{\text {nd }}$ and $4^{\text {th }}$, but no statistically significant difference was observed at week $12^{\text {th }}$ after the procedure. There were also no statistically significant differences in pain perception after surgery, visual acuity, and corneal clarity in the 12 months after the procedure. Recurrent corneal epithelial defects have also not been observed in patients after CXL, which occurred in $12 \%$ of patients [51]. Overall, CXL can be considered a short-term benefit method in patients with bullous keratopathy. CXL can be recommended particularly for people who do not want or cannot undergo endothelial keratoplasty.

\section{CROSS-LINKING TECHNIQUE}

Generally, during the procedure, the patient takes a prone position under local drip anesthesia. The corneal epithelium is removed (epi-off), and alcohol can be used, similarly to the LASEK method. Alternatively, some researchers propose to use transepithelial CXL without removing the epithelium (epi-on). It is known that the epi-on is preferred by patients. In the case of the transepithelial procedures, 
there are issues with poor penetration of riboflavin into the essence of the cornea, which may be supported by iontophoresis or chemical factors [52].

Even though this is a controversial topic, it cannot be conclusively stated at present that epi-on CXL is as effective as epi-off [52]. The cornea is then soaked in riboflavin and irradiated with $370 \mathrm{~nm}$ UVA light. The soaking in riboflavin is repeated every 2-3 minutes. In a standard protocol, energy of $3 \mathrm{~mW} / \mathrm{cm}^{2}$ is used for 30 minutes, and in an accelerated protocol, energy of $7 \mathrm{~mW} / \mathrm{cm}^{2}$ is used for 15 minutes, $9 \mathrm{~mW} / \mathrm{cm}^{2}$ for 10 minutes, and $18 \mathrm{~mW} / \mathrm{cm}^{2}$ for 5 minutes. The laboratory results for the accelerated CXL protocols were encouraging $[53,54]$, but it cannot be conclusively stated that these protocols are as effective as the standard protocol.

What is new about the cross-linking technique is that it can be used in a sitting position, by fixing the UVA lamp to the slit lamp. The potential benefits of such an application are extensive. First, there is no need for an operating room, so the procedure can be carried out in smaller centers and ophthalmic practices. This increased access to CXL is especially vital in case of developing countries, where access to ordinary ophthalmological practices may require several hours of traveling, and even more to reach a treatment room. Moreover, the device is easy to use, small and portable. A slit lamp can also be considered a natural working environment for an ophthalmologist (fig. 1).

\section{CONCLUSIONS}

Clinical trials showed that CXL is an effective method of reducing the progression of corneal ectasia. With this method we can avoid the need for more invasive procedures, such as corneal transplantation, or postpone them. Scientific reports have also indicated the effectiveness of CXL in the treatment of bacterial infections of the cornea and bullous keratopathy.

\section{Figures: from the author's own materials.}

\section{FIGURE ( 1}

Cross-linking performed with a slit lamp. After removal of the epithelium and application of riboflavin into the cornea, the cross-linking of the cornea is performed using a UVA lamp (C-Eye, EMAGine AG, Zug, Switzerland) mounted on a Haag Streit slit lamp.

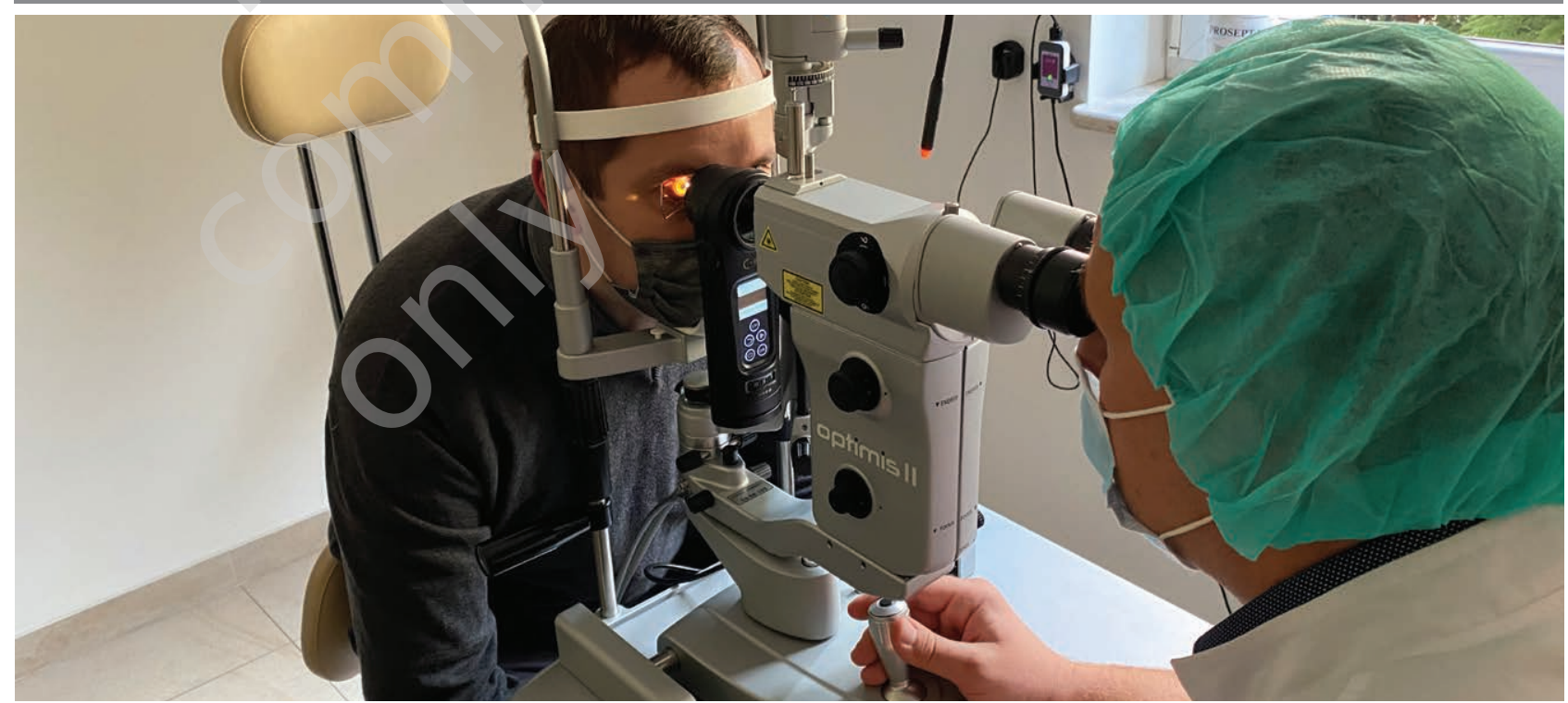

\section{CORRESPONDENCE}

\section{Piotr Kanclerz, MD, PhD}

Hygeia Clinic, Gdańsk

80-286 Gdańsk, ul. Jaśkowa Dolina 57

phone: +4858776 40 46; fax: +48587764046

e-mail: p.kanclerz@gumed.edu.pl

\section{ORCID}

Piotr Kanclerz - ID - http://orcid.org/0000-0002-8036-7691 Katarzyna Przewłócka - ID - http://orcid.org/0000-0003-0736-6008 


\section{References}

1. Wollensak G. Crosslinking treatment of progressive keratoconus: new hope. Curr Opin Ophthalmol. 2006; 17: 356-60.

2. Sherwin T, Brookes NH. Morphological changes in keratoconus: pathology or pathogenesis. Clin Exp Ophthalmol. 2004; 32: 211-7. http://doi.org/10.1111/j.1442-9071.2004.00805.x.

3. Wollensak G, Spörl E, Reber F et al. Corneal Endothelial Cytotoxicity of Riboflavin/UVA Treatment in vitro. Ophthalmic Res. 2003; 35: 324-8. http://doi.org/10.1159/000074071.

4. Spoerl E, Mrochen M, Sliney D et al. Safety of UVA-riboflavin cross-linking of the cornea. Cornea. 2007; 26: 385-9.

5. Hafezi F, Mrochen M, Iseli HP et al. Collagen crosslinking with ultraviolet-A and hypoosmolar riboflavin solution in thin corneas. J Cataract Refract Surg. 2009; 35: 621-4. http://doi.org/10.1016/j.jcrs.2008.10.060.

6. Wollensak G, Spoerl E, Seiler T. Riboflavin/ultraviolet-a-induced collagen crosslinking for the treatment of keratoconus. Am J Ophthalmol. 2003; 135: 620-7.

7. Caporossi A, Baiocchi S, Mazzotta C et al. Parasurgical therapy for keratoconus by riboflavin-ultraviolet type A rays induced cross-linking of corneal collagen. J Cataract Refract Surg. 2006; 32: 837-45. http://doi.org/10.1016/j.jcrs.2006.01.091.

8. Caporossi A, Mazzotta C, Baiocchi S et al. Long-term Results of Riboflavin Ultraviolet A Corneal Collagen Cross-linking for Keratoconus in Italy: The Siena Eye Cross Study. Am J Ophthalmol. 2010; 149: 585-93. http://doi.org/10.1016/j.ajo.2009.10.021.

9. Asri $D$, Touboul $D$, Fournié $P$ et al. Corneal collagen crosslinking in progressive keratoconus: Multicenter results from the French National Reference Center for Keratoconus. J Cataract Refract Surg. 2011; 37: 2137-43. http://doi.org/10.1016/j.jcrs.2011.08.026.

10. Kymionis GD, Mikropoulos DG, Portaliou DM et al. An overview of corneal collagen cross-linking (CXL). Adv Ther. 2013; 30: 858-69.

11. Hafezi F, Iseli HP. Pregnancy-related exacerbation of iatrogenic keratectasia despite corneal collagen crosslinking. J Cataract Refract Surg. 2008; 34: 1219-21.

12. Brancati F. A locus for autosomal dominant keratoconus maps to human chromosome 3p14-q13. J Med Genet. 2004; 41: 188-92. http:// doi.org/10.1136/jmg.2003.012872.

13. Rabinowitz YS. Keratoconus. Surv Ophthalmol. 1998; 42: 297-319.

14. Asimellis G, Kaufman EJ. Keratoconus. StatPearls, Treasure Island (FL): StatPearls Publishing; 2020.

15. Ferdi AC, Nguyen V, Gore DM et al. Keratoconus Natural Progression: A Systematic Review and Meta-analysis of 11529 Eyes. Ophthalmology. 2019; 126: 935-45.

16. Torres Netto EA, Al-Otaibi WM, Hafezi NL et al. Prevalence of keratoconus in paediatric patients in Riyadh, Saudi Arabia. Br J Ophthalmol. 2018; 102: 1436-41.

17. Gokul A, Patel DV, Watters GA et al. The natural history of corneal topographic progression of keratoconus after age 30 years in non-contact lens wearers. Br J Ophthalmol. 2017; 101: 839-44.

18. Piñero DP, Alio JL, Tomás J et al. Vector analysis of evolutive corneal astigmatic changes in keratoconus. Invest Ophthalmol Vis Sci. 2011; 52: 4054-62.

19. Walker RN, Khachikian SS, Belin MW. Scheimpflug photographic diagnosis of pellucid marginal degeneration. Cornea. 2008; 27: 963-6.

20. Krachmer JH. Pellucid marginal corneal degeneration. Arch Ophthalmol. 1978; 96: 1217-21.

21. Rodrigues MM, Newsome DA, Krachmer JH et al. Pellucid marginal corneal degeneration: a clinicopathologic study of two cases. Exp Eye Res. 1981; 33: 277-88.

22. Rodriguez-Prats J, Galal A, Garcia-Lledo M et al. Intracorneal rings for the correction of pellucid marginal degeneration. J Cataract Refract Surg. 2003; 29: 1421-4.

23. Sahu J, Raizada K. Pellucid Marginal Corneal Degeneration. StatPearls, Treasure Island (FL): StatPearls Publishing; 2020.

24. Wollensak G. Corneal collagen crosslinking: new horizons. Expert Rev Ophthalmol. 2010; 5: 201-15. http://doi.org/10.1586/eop.10.7.

25. Spadea L. Corneal Collagen Cross-Linking with Riboflavin and UVA Irradiation in Pellucid Marginal Degeneration. J Refract Surg. 2010; 26: 375-7. http://doi.org/10.3928/1081597x-20100114-03.

26. Stojanovic A, Zhang J, Chen X et al. Topography-Guided Transepithelial Surface Ablation Followed by Corneal Collagen Cross-Linking Performed in a Single Combined Procedure. J Refract Surg. 2010; 26: 145-52. http://doi.org/10.3928/1081597x-20100121-10.

27. Kymionis GD, Karavitaki AE, Kounis GA et al. Management of pellucid marginal corneal degeneration with simultaneous customized photorefractive keratectomy and collagen crosslinking. J Cataract Refract Surg. 2009; 35: 1298-301.

28. Hassan Z, Nemeth G, Modis L et al. Collagen cross-linking in the treatment of pellucid marginal degeneration. Indian J Ophthalmol. 2014; 62: 367-70.

29. Rao SN, Epstein RJ. Early onset ectasia following laser in situ keratomileusus: case report and literature review. J Refract Surg. 2002; 18: 177-84.

30. Giri P, Azar DT. Risk profiles of ectasia after keratorefractive surgery. Curr Opin Ophthalmol. 2017; 28: 337-42.

31. Randleman JB, Woodward M, Lynn MJ et al. Risk assessment for ectasia after corneal refractive surgery. Ophthalmology. 2008; 115: 37-50. 
32. Binder PS. Ectasia after laser in situ keratomileusis. J Cataract Refract Surg. 2003; 29: 2419-29. http://doi.org/10.1016/j.jcrs.2003.10.012.

33. O'Keefe M, Kirwan C. Laser epithelial keratomileusis in 2010 - a review. Clin Exp Ophthalmol. 2010; 38: 183-91. http://doi.org/10.1111/ j.1442-9071.2010.02198.x.

34. Brenner LF, Alió JL, Vega-Estrada A et al. Indications for intrastromal corneal ring segments in ectasia after laser in situ keratomileusis. J Cataract Refract Surg. 2012; 38: 2117-24.

35. Tatar MG, Aylin Kantarci F, Yildirim A et al. Risk Factors in Post-LASIK Corneal Ectasia. J Ophthalmol. 2014; 2014: 204191.

36. Kanclerz P, Khoramnia R, Wang X. Current Developments in Corneal Topography and Tomography. Diagnostics. 2021; 11: 1466. http:// doi.org/10.3390/diagnostics11081466.

37. Hafezi F, Kanellopoulos J, Wiltfang R et al. Corneal collagen crosslinking with riboflavin and ultraviolet A to treat induced keratectasia after laser in situ keratomileusis. J Cataract Refract Surg. 2007; 33: 2035-40. http://doi.org/10.1016/j.jcrs.2007.07.028.

38. Garg P, Das S, Roy A. Collagen Cross-linking for Microbial Keratitis. Middle East Afr J Ophthalmol. 2017; $24: 18-23$.

39. Iseli HP, Thiel MA, Hafezi F et al. Ultraviolet A/riboflavin corneal cross-linking for infectious keratitis associated with corneal melts. Cornea. 2008; 27: 590-4.

40. Sorkhabi R, Sedgipoor M, Mahdavifard A. Collagen cross-linking for resistant corneal ulcer. Int Ophthalmol. 2013; 33: 61-6.

41. Makdoumi K, Mortensen J, Crafoord S. Infectious keratitis treated with corneal crosslinking. Cornea. 2010; 29: 1353-8.

42. Shetty R, Nagaraja H, Jayadev C et al. Collagen crosslinking in the management of advanced non-resolving microbial keratitis. Br J Ophthalmol. 2014; 98: 1033-5. http://doi.org/10.1136/bjophthalmol-2014-304944.

43. Feizi S, Karimian F, Esfandiari H. Corneal crosslinking for the treatment of infectious keratitis: a review. Expert Rev Ophthalmol. 2021; 16: 287-95.

44. Papaioannou L, Miligkos M, Papathanassiou M. Corneal Collagen Cross-Linking for Infectious Keratitis: A Systematic Review and Meta-Analysis. Cornea. 2016; 35: 62-71.

45. Morishige N, Sonoda KH. Bullous keratopathy as a progressive disease: evidence from clinical and laboratory imaging studies. Cornea. 2013; 32(suppl 1): S77-83.

46. Gonçalves ED, Campos M, Paris F et al. [Bullous keratopathy: etiopathogenesis and treatment]. Arq Bras Oftalmol. 2008; 71: 61-4.

47. Pot SA, Gallhöfer NS, Walser-Reinhardt L et al. Treatment of bullous keratopathy with corneal collagen cross-linking in two dogs. Vet Ophthalmol. 2015; 18: 168-73.

48. Ghanem RC, Santhiago MR, Berti TB et al. Collagen crosslinking with riboflavin and ultraviolet-A in eyes with pseudophakic bullous keratopathy. J Cataract Refract Surg. 2010; 36: 273-6. http://doi.org/10.1016/j.jcrs.2009.07.041.

49. Krueger RR, Ramos-Esteban JC, Kanellopoulos AJ. Staged Intrastromal Delivery of Riboflavin With UVA Cross-linking in Advanced Bullous Keratopathy: Laboratory Investigation and First Clinical Case. J Refract Surg. 2008; 24(7): S730-6. http://doi. org/10.3928/1081597x-20080901-17.

50. Wollensak G, Aurich H, Wirbelauer C et al. Potential use of riboflavin/UVA cross-linking in bullous keratopathy. Ophthalmic Res. 2009; 41: 114-7.

51. Choy BNK, Ng ALK, Zhu MM et al. Randomized Control Trial on the Effectiveness of Collagen Cross-linking on Bullous Keratopathy. Cornea. 2020; 39: 1341-7.

52. O'Brart DPS. Corneal collagen crosslinking for corneal ectasias: a review. Eur J Ophthalmol. 2017; 27: 253-69.

53. Wernli J, Schumacher S, Spoerl E et al. The Efficacy of Corneal Cross-Linking Shows a Sudden Decrease with Very High Intensity UV Light and Short Treatment Time. Invest Opthalmol Vis Sci. 2013; 54: 1176. http://doi.org/10.1167/iovs.12-11409.

54. Aldahlawi NH, Hayes S, O'Brart DPS et al. Standard versus accelerated riboflavin-ultraviolet corneal collagen crosslinking: Resistance against enzymatic digestion. J Cataract Refract Surg. 2015; 41: 1989-96. http://doi.org/10.1016/j.jcrs.2015.10.004. 
Authors' contributions:

All authors contributed equally to the article.

Conflict of interest:

None.

Financial support:

None.

Ethics:

The content presented in the article complies with the principles of the Helsinki

Declaration, EU directives and harmonized requirements for biomedical journals.

c) Medical Education. For private and non-commercial use only. Downloaded from https://www.journalsmededu.pl/index.php/ophthatherapy/index: 26.04.2023; 15:18,22 